\title{
The Imperfect Fibonacci and Lucas Numbers
}

\author{
JOHN H. JAROMA
}

\begin{abstract}
A perfect number is any positive integer that is equal to the sum of its proper divisors. Several years ago, F. Luca showed that the Fibonacci and Lucas numbers contain no perfect numbers. In this paper, we alter the argument given by Luca for the nonexistence of both odd perfect Fibonacci and Lucas numbers, by making use of an 1888 result of C. Servais. We also provide a brief historical account of the study of odd perfect numbers.
\end{abstract}

\section{INTRODUCTION}

It has been shown as sufficient by Euclid and as necessary by Euler that an even number is perfect if and only if it is equal to

$$
2^{p-1}\left(2^{p}-1\right)
$$

where $2^{p}-1$ is prime. Primes of the form $2^{p}-1$ are called Mersenne primes. They are named in honor of the 17th century priest, Fr. M. Mersenne (1588-1648), who claimed that such numbers are prime provided that $p \in\{2,3,5,7,13,17,19,31,67,127,257\}$ and are composite for all other values of $p \leq 257$. Although Mersenne's conjecture contained five mistakes, it had taken more than 300 years for mathematicians to discover them all.

Because of the Euclid-Euler defining characteristic of an even perfect number, the discovery of a new Mersenne prime is equivalent to the finding of a new even perfect number. As of 2008, only 44 Mersenne primes have been discovered. The four smallest were known at the time of Euclid. The prevailing conjecture is that there are infinitely many.

An equally, if not even more celebrated open problem is the question of whether or not an odd perfect number exists. It has remained unanswered for over two millennia. Nevertheless, a significant step toward a better understanding of them occurred in the 18th century 
when L. Euler provided us with their canonical form. In particular, he showed that if $n$ is an odd perfect number, then it necessarily follows that

$$
n=p^{\alpha} q_{1}^{2 \beta_{1}} q_{2}^{2 \beta_{2}} \cdots q_{k}^{2 \beta_{k}}
$$

where, $p, q_{1}, q_{2}, \ldots q_{k}$, are distinct odd primes and $p \equiv \alpha \equiv 1(\bmod 4)$.

In his 1972 Ph.D. thesis, C. Pomerance asserted that the modern era of research on odd perfect numbers began with J. J. Sylvester [33], for in 1888 Sylvester published a series of papers that further qualified the structure that an odd perfect number must assume. Specifically, he demonstrated that such a number has at least four distinct prime divisors. Sylvester also established a lower bound of eight on the number of distinct prime factors that an odd perfect number can have provided that it is not divisible by three [43]. In addition, he showed that no odd perfect number is divisible by 105 [43]. Furthermore, before that year was over, Sylvester also improved the unrestricted bound on the number of distinct prime divisors of an odd perfect to five [44].

Sylvester offered the reader some of his thoughts regarding the existence of an odd perfect number in [42] when he equated the question to a problem of the ages comparable in difficulty to that which previously to the labours of Hermite and Lindemann ... environed the subject of the quadrature of the circle. He contended that odd perfect numbers do not exist and in [41] declared that ... a prolonged meditation on the subject has satisfied me that the existence of any one such - its escape, so to say, from the complex web of conditions which hem it in on all sides - would be little short of a miracle.

Recently, Luca showed that perfect numbers do not exist among either the Fibonacci numbers $\left\{F_{n}\right\}=\{1,1,2,3,5,8,13,21,34,55, \ldots\}$ or the Lucas numbers $\left\{L_{n}\right\}=\{1,3,4,7,11,18,29,47,76,123$, $\ldots\}$ [23]. His argument for the odd perfect number showed that if either $F_{n}$ or $L_{n}$ is odd perfect, then $n=p$, where $p$ an odd prime. He then proved that $F_{p}$ is not an odd perfect number by quoting an earlier result of his that asserts $\sigma\left(F_{n}\right) \leq F_{\sigma(n)}, \forall n \geq 1[24] .^{1}$ For the case of the Lucas numbers, Luca demonstrated that $\sigma\left(L_{p}\right)<2 L_{p}$, for all primes $p \geq 2$.

\footnotetext{
${ }^{1}$ If we let $n$ be any positive integer, then $\sigma(n)$ denotes the sum of the positive divisors of $n$.
} 
We later summarize Luca's argument for the nonexistence of an even perfect Lucas number, as well as show there are no even perfect Fibonacci numbers by recapping the solution of [31]. Upon doing this, we offer a proof, apart from the one given by Lucas, demonstrating the impossibility of either an odd perfect Fibonacci or Lucas number. Our main tool will be an 1888 theorem of C. Servais that places an upper bound on the least prime divisor of an odd perfect number [38].

Before this is accomplished, we present the following account of the study of odd perfect numbers.

\section{Brief Study of Odd Perfect Numbers}

This section briefly recaps and updates the history of the study of odd perfect numbers offered in [9].

Approximately twenty three hundred years ago, Euclid showed in Proposition 36 of Book IX of his Elements that a number of the form $2^{n-1}\left(2^{n}-1\right)$ is perfect provided that $2^{n}-1$ is prime. Four hundred years later, Nicomachus of Gerasa continued the study of perfect numbers in his Introductio Arithmetica. Unfortunately, all of his assertions, including the declaration that all perfect numbers are even, were given without proof. Nonetheless, his conjectures were taken as fact for centuries.

It appears that the first mathematician of note to suggest that an odd perfect number exists was R. Descartes. In a letter to Mersenne dated November 15, 1638, he announced that he could demonstrate that every odd perfect number must be of the form $p s^{2}$, where $p$ is a prime. Furthermore, he stated that he saw no reason to prevent the existence of an odd perfect number and cited the example of $p=22021$ and $s=3 \cdot 7 \cdot 11 \cdot 13$ as evidence. For, $p s^{2}$ would be an odd perfect number provided one pretends that 22021 is prime.

In 1832, B. Peirce studied existence criteria from a different perspective by establishing a lower bound of four on the number of distinct prime divisors that an odd perfect number can have [32]. We remark that the credit for this important discovery seems to have eluded Peirce, being often misdirected to either Sylvester [43] or to Servais [39]. Both of these mathematicians independently proved the same result more than fifty years later (See [43] and [39].) In fact, even L. E. Dickson neglected to credit Peirce with this important discovery in his magnum opus, History of the Theory Numbers [7]. 
However, as noted earlier, Sylvester did break new ground in 1888 by improving the said lower bound to five [44]. The year 1888 also saw Servais placing an upper bound of $k+1$ on the least prime divisor of an odd perfect number with $k$ distinct prime divisors [38].

In 1913, Dickson demonstrated that for any integral value of $k$, there are only finitely many odd perfect numbers with $k$ components ${ }^{2}$ [8]. He proved this as a corollary to a similar result for odd primitive non-deficient numbers. By definition, such numbers necessarily contain all the odd perfect numbers ${ }^{3}$. A significant aspect of Dickson's paper is that one may now conduct a search for the an odd perfect number with $k$ components by initially listing out all of the finitely many primitive odd non-deficient numbers associated with that $k$ value and then checking for those among them are equal to the sum of their proper divisors. Alas, the approach is not feasible for most values of $k$, for the resulting lists quickly become intractably large.

In 1925, I. Gradstein advanced the lower bound on the number of distinct prime divisors on an odd perfect number to six [11]. In 1949, H. J. Kanold revisited the four-divisor case and published a proof of the same result given in earlier years by Peirce, Servais, Sylvester, and Dickson [27]. The significance of this paper extended considerably beyond the stated result for in it, Kanold demonstrated that the largest prime divisor of an odd perfect number must exceed 60. This marked the first theorem of its kind. Moreover, it represented the initial contribution to a class of propositions that would ultimately be suggested by Pomerance some twenty-five years later.

More specifically, because the approach to the odd perfect number question from the perspective of Dickson's paper is impractical, it was necessary to seek out alternative approaches to studying the structure of an odd perfect number. In 1974, and in addition to showing that an odd perfect number must have at least seven distinct prime divisors ${ }^{4}$, Pomerance proposed a class of theorems for consideration: An odd perfect number is divisible by $j$ distinct primes $>N[33]$.

Furthermore, along this line of thought, Kanold's 1949 result of $j=1$ and $N=60$ was improved for the $j=1$ case to $N=11200$

\footnotetext{
${ }^{2}$ For example, that the components of (1) are $p^{\alpha}, q_{1}^{2 \beta_{1}}, q_{2}^{2 \beta_{2}}, \ldots q_{k}^{2 \beta_{k}}$.

${ }^{3} \mathrm{~A}$ deficient number is any integer $n$ with $\sigma(n)<2 n$. So, $n$ is a non-deficient number if $\sigma(n) \geq 2 n$. Dickson had called a number primitive non-deficient provided that it is not a multiple of a smaller non-deficient number.

${ }^{4}$ In 1974 , N. Robbins independently proved the same result [36].
} 
by P. Hagis, Jr. and W. McDaniel [15] in 1973. Two years later, they bettered their own result by exhibiting $N=100110$ [16]. In 1975, Pomerance became the first to illustrate a case for $j>1$ upon showing $j=2$ and $N=138$ [34].

In 2008, T. Goto and Y. Ohno showed that an odd perfect number must have a factor greater than $10^{8}$. This improved the 2003 result of P. Jenkins who demonstrated that the largest prime divisor of an odd perfect number exceeds $10^{7}$ [22], which augmented the earlier lower bound of $10^{6}$ discovered by Hagis and G. Cohen in 1998 [4].

In 1999 and 2000, D. Iannucci published initial results on the second and third largest prime divisors of an odd perfect number. He proved they exceed 10000 and 100, respectively [19], [20]. It appears that these remain as the best such estimates to date.

The study of odd perfect numbers has also included attempts to provide a bound on its magnitude. In 1908, A. Turăninov established a lower bound of 2000000 . The current best estimate of this kind has been given by R. P. Brent, Cohen, and H. J. J. te Riele in 1991 [1], which showed that any odd perfect number is necessarily greater than $10^{300}$. This result was achieved by developing an algorithm which demonstrated that if there exists an odd perfect number $n$ then $n>K$, upon which they applied the algorithm to $K=10^{300}$.

In 1994, R. Heath-Brown proved that if $n$ is odd and $\sigma(n)=a n$, then $n<(4 d)^{4^{k}}$, where $d$ is the denominator in $a$ and $k$ is the number of distinct prime factors of $n$ [18]. In particular, if $n$ is an odd perfect number then $n$ has an upper bound of $4^{4^{k}}$. This represents an improvement over the previous best estimate of $n<$ $(4 k)^{(4 k)^{2^{k^{2}}}}$, which was given by Pomerance in 1977 [35]. HeathBrown has remarked that his bound is still too large to be of practical value. Nevertheless, we remark that when his bound is viewed in conjunction with the lower bound of $10^{300}$, Sylvester's result that every odd perfect number has at least five distinct divisors follows immediately; that is, $10^{300}<n<4^{4^{k}}$ implies $k>4$.48. In 1999, R. J. Cook improved Heath-Brown's result to $n<(2.124)^{4^{k}}$ [6]. In 2003, P. Nielsen further reduced it to $2^{4^{k}}[29]$.

Presently, the best result for the least number of distinct prime divisors that an odd perfect number can have is nine. This was recently discovered by Nielsen [30]. It is a long-awaited improvement over the bound of eight established independently in 1979 by E. Z. Chien [3] (who published nothing of his work) and by Hagis 
in 1980 [12]. Hagis's original proof contained almost two hundred manuscript pages. We note that Cohen and Sorli in 2003 described an algorithmic approach for showing that if there exists an odd perfect number, then it has $t$ distinct prime factors [5]. In that work, they also discussed the algorithm's applicability to the case $t \geq 9$. Nielsen's demonstration ultimately avoids previous computational results for odd perfect numbers.

Some obtained estimated on the total number of prime divisors that an odd perfect number can have. In 1986, M. Sayers showed that such a number necessarily has at least 29 such factors [37]. This was later improved to 37 by Iannucci and Sorli [21]. The best estimate to date appears to be 75 , given recently by Hare [17].

The best improvement to Sylvester's bound on the number of distinct prime factors of an odd perfect number not divisible by three now stands at twelve which also appears in Nielsen's paper [30]. The best previous estimate of eleven had been obtained independently in 1983 by both Hagis [13] and M. Kishore [28].

Finally, we point out that a study of odd perfect numbers from a somewhat different perspective was initiated in 1937 by R. Steuerwald upon showing that not all the $\beta_{i}$ 's in Euler's canonical form given by (1) can all be equal to one. This continued in 1941 when Kanold showed that neither may all of the $\beta_{i}$ 's be equal to two nor may one of the $\beta_{i}$ 's be equal to two while all the rest are equal to one [26]. In 1972, Hagis and McDaniel proved in [14] that not all the $\beta_{i}$ can be equal to three. In 2003, Iannucci and Sorli showed that an odd perfect number cannot be divisible by three if for all $i, \beta_{i} \equiv 2$ $(\bmod 3)$ or $\beta_{i} \equiv 2(\bmod 5)[\mathbf{2 1}]$.

\section{3. $F_{n}$ AND $L_{n}$ ARE NOt Even PeRfect}

In this section, we present previously developed arguments for the nonexistence of even perfect Fibonacci and Lucas numbers. As previously noted, if $2^{p}-1$ is prime, then a necessary and sufficient condition for an even number $N$ to be perfect is that it is necessarily of the form

$$
N=2^{p-1}\left(2^{p}-1\right) .
$$

Now, to show that a Fibonacci number cannot be even perfect, we refer Padwa's 1972 solution of the problem posed by R. Whitney [31]: Prove that there are no even perfect Fibonacci numbers. For the Lucas numbers, we illustrate Luca's proof given in [23]. 
Theorem 1. There are no even perfect Fibonacci or Lucas numbers.

Proof. Let $N=2^{p-1}\left(2^{p}-1\right)$ be a perfect number.

Case 1. (Whitney/Padwa) Assume that $N$ is a Fibonacci number. Since all even perfect numbers are given according to (2), this implies that all even perfect numbers greater than 28 are also divisible by 16 . Now, the only Fibonacci numbers divisible by 16 are also divisible by 9 . Thus, a Fibonacci number cannot be of the form $2^{p-1}\left(2^{p}-1\right)$. Therefore, no Fibonacci number is even perfect.

Case 2. (Luca) Assume that $N$ is a Lucas number. First, if $p=2$ then $N=6$, and, if $p=3$ then $N=28$. Since both of these are not Lucas numbers, it is without loss of generality that we assume $p>3$. In light of (2), this implies that $8 \mid L_{k}$. However, this is impossible, as no Lucas number is divisible by 8 . Therefore, there are no even perfect Lucas numbers.

\section{Generating $F_{n}$ And $L_{n}$ From the Lucas Sequences}

Before we proceed with our demonstration that $F_{n}$ and $L_{n}$ cannot be odd perfect numbers, we will need to view these numbers as iterations of a specific Lucas and companion Lucas sequence, respectively.

To this end, let $P$ and $Q$ be relatively prime integers. The Lucas sequences are defined recursively by

$U_{n+2}(P, Q)=P U_{n+1}-Q U_{n}, U_{0}=0, U_{1}=1, n \in\{0,1, \ldots\}$.

Similarly, the companion Lucas sequences are

$$
V_{n+2}(P, Q)=P V_{n+1}-Q V_{n}, V_{0}=2, V_{1}=P, n \in\{0,1, \ldots\} .
$$

We point out that the Fibonacci numbers, $\left\{F_{n}\right\}$, are produced by the Lucas sequence $\left\{U_{n}(1,-1)\right\}$ and the Lucas numbers $\left\{L_{n}\right\}$ are generated by the companion Lucas sequence $\left\{V_{n}(1,-1)\right\}$. Furthermore, since (3) and (4) are linear they are solvable. In particular, for $n \in\{0,1, \ldots\}$,

$$
\begin{gathered}
F_{n}=U_{n}(1,-1)=\frac{1}{\sqrt{5}}\left[\left(\frac{1+\sqrt{5}}{2}\right)^{n}-\left(\frac{1-\sqrt{5}}{2}\right)^{n}\right] ; \\
L_{n}=V_{n}(1,-1)=\left(\frac{1+\sqrt{5}}{2}\right)^{n}+\left(\frac{1-\sqrt{5}}{2}\right)^{n} .
\end{gathered}
$$




\section{5. $F_{n}$ ANd $L_{n}$ ARE NOt Odd Perfect}

Our proof of the nonexistence of Fibonacci and Lucas odd perfect numbers relies on the following proposition of Servais which places an upper bound on the least prime divisor of an odd perfect number [38].

Theorem 2. (Servais) The least prime divisor of an odd perfect number with $k$ distinct prime factors does not exceed $k$.

The next result demonstrated by Luca in [23] will also be utilized in our argument.

Lemma 1. Let either $F_{n}$ or $L_{n}$ be an odd perfect number. Then, $n$ is prime.

In a given Lucas sequence, the rank of apparition of $p$ is the index of the first term that contains $p$ as a divisor. A prime factor of either $U_{n}$ or $V_{n}$ is primitive provided that its rank of apparition is $n$. Such a factor is called intrinsic if it divides $n$. Otherwise, it is said to be extrinsic.

The following three lemmas come from either [2] or [25].

Lemma 2. The odd extrinsic factors of $U_{n}$ are of the form $r n \pm 1$.

Lemma 3. The odd extrinsic factors of $V_{n}$ are of the form $2 k n \pm 1$.

Lemma 4. Assume that $p \nmid P Q$ and let $\omega$ denote the rank of apparition of an odd prime $p$ in the sequence $\left\{U_{n}(P, Q)\right\}$. Then, $p \mid U_{n}$ if and only if $n=k \omega$.

Lemma 5 is based on results found in [2].

Lemma 5. For any $p \neq 5$, both $p \nmid F_{p}$ and $p \nmid L_{p}$.

We are now ready to offer a proof of the nonexistence of odd perfect Fibonacci numbers and odd perfect Lucas numbers. Both cases are demonstrated at once.

Theorem 3. There are no odd perfect Fibonacci or Lucas numbers.

Proof. By Lemma 1 and the fact that $F_{2}=1, L_{2}=3, F_{5}=5$, and $L_{5}=11$ are all not perfect, it suffices to consider only the case where $n=p$ is an odd prime not equal to 5 . Now, for the sake of contradiction, let's assume that there exists a prime $p$ for 
which either $F_{p}\left(L_{p}\right)$ is an odd perfect number. Let $d$ be the least prime divisor of $F_{p}\left(L_{p}\right)$. Since the index $p$ of $F_{p}\left(L_{p}\right)$ is prime, it follows from Lemma 4 that $d$ is a primitive prime factor of that term. Furthermore, since $p \neq 5$, it follows by Lemma 5 that every prime factor of $F_{p}\left(L_{p}\right)$ is extrinsic. As $F_{3}$ and $L_{3}$ are respectively, the only even Fibonacci and Lucas numbers of prime index, we conclude that for all $p>3$, every primitive factor of $F_{p}\left(L_{p}\right)$ is odd. Since $d$ and $p$ are odd, it then follows from Lemma 2 and Lemma 3 that $d=r p \pm 1=2 k p \pm 1$. Hence, $d \geq 2 p-1$. Moreover, Theorem 2 tells us that $F_{p}\left(L_{p}\right)$ has at least $2 p-1$ distinct prime factors. Therefore, utilizing (5), (6), and Lemma 3 , we obtain

$$
\begin{aligned}
2\left(\frac{1+\sqrt{5}}{2}\right)^{2 p-1}> & \left(\frac{1+\sqrt{5}}{2}\right)^{2 p-1}+\left(\frac{1-\sqrt{5}}{2}\right)^{2 p-1}=L_{2 p-1}>L_{p} \geq \\
\geq & (2 p-1)(2 p+1)(4 p-1)(4 p+1) \\
& \quad \ldots[2(2 p-2)-1][2(2 p-2)+1][2(2 p-1)-1],
\end{aligned}
$$

where the last product consists of $2 p-1$ terms, which for all $p$, is impossible.

\section{REFERENCES}

[1] R. P. Brent, G. L. Cohen and H. J. J. te Riele, Improved techniques for lower bounds for odd perfect numbers, Math. of Comp. 57 (1991), 857-868.

[2] R. D. Carmichael, On the numerical factors of the arithmetic forms $\alpha^{n} \pm \beta^{n}$, Annals of Mathematics, (2) 15 (1913-14), 30-70.

[3] E. Z. Chien, An odd perfect number has at least eight prime factors, Ph.D. Thesis, Pennsylvania State University, (1979).

[4] G. Cohen and P. Hagis Jr., Every odd perfect number has a prime factor that exceeds $10^{6}$, Math. of Comp. 67 (1998), 1323-1330.

[5] G. Cohen and R. Sorli, On the number of distinct prime factors of an odd perfect number, J. Discrete Algorithms 1 (2003), 21-35.

[6] R. J. Cook, Bounds for odd perfect numbers, CRM Proc. and Lect. Notes, Centres de Recherches Mathématiques 19 (1999), 67-71.

[7] L. E. Dickson, History of the theory of numbers, Vol I Washington: Carnegie Institute of Washington. 1919-1923; reprint ed., Chelsea Publ. Co., New York (1952).

[8] L. E. Dickson, Finiteness of the odd perfect and primitive abundant numbers with $n$ distinct prime factors, Amer. J. Math. 35 (1913), 413-422.

[9] S. Gimbel and J. H. Jaroma, Sylvester: Ushering in the modern era of research on odd perfect numbers, Int.: Elec. J. Comb. Num. Th. 3 (2003), A16.

[10] T. Goto and Y. Ohno, Odd perfect numbers have a factor exceeding $10^{8}$, Math. of Comp., 77 (2008), 1859-1868. 
[11] I. S. Gradstein, O nečetnych soveršennych čislah., Mat. Sb. 32 (1925), 476510.

[12] P. Hagis Jr., Outline of a proof that every odd perfect number has at least eight prime factors, Math. of Comp. (1980), 1027-1032.

[13] P. Hagis Jr., Sketch of a proof that every odd perfect number relatively prime to 3 has at least eleven prime factors, Math. of Comp. 40 (1983), 399-404.

[14] P. Hagis Jr. and W. McDaniel, A new result concerning the structure of odd perfect numbers, Proc. Am. Math. Soc. 32 (1972), 13-15.

[15] P. Hagis Jr. and W. McDaniel, On the largest prime divisor of an odd perfect number, Math. of Comp. 27 (1973), 955-957.

[16] P. Hagis Jr. and W. McDaniel, On the largest prime divisor of an odd perfect number, Math. of Comp. 29 (1975), 922-924.

[17] K. Hare, New techniques for bounds on the total number of prime factors of an odd perfect number, Math. of Comp. 74 (2005), 1003-1008.

[18] D. R. Heath-Brown, Odd perfect numbers, Math. Proc. Camb. Phil. Soc. 115 (1994), 191-196.

[19] D. E. Iannucci, The second largest divisor of an odd perfect number exceeds ten thousand, Math. of Comp. 68 (1999), 1749-1760.

[20] D. E. Iannucci, The third largest divisor of an odd perfect number exceeds one hundred, Math. of Comp. 69 (2000), 867-879.

[21] D. E. Iannucci and R. M. Sorli, On the total number of prime factors of an odd perfect number, Math. of Comp. 72 (2003), 1549-1554.

[22] P. M. Jenkins, The largest prime divisor of an odd perfect number exceeds 10,000,000, Math. of Comp. 72 (2003), 1549-1554.

[23] F. Luca, Perfect Fibonacci and Lucas numbers, Rend. Cir. Mat. Palermo (Serie II) XLIX (2000), 313-318.

[24] F. Luca, Arithmetic functions of Fibonacci and Lucas numbers, Fib. Qtly. 37 (1999), 265-268.

[25] É. Lucas, Théorie des fonctions numériques simplement périodiques, American J. Math. 1 (1878) 184-240, 289-321.

[26] H. J. Kanold, Untersuchungen über ungerade vollkommene Zahlen, J. Reine Angew. Math. 183 (1941), 98-109.

[27] H. J. Kanold, Folgerungen aus dem Vorkommen einer Gauss'schen Primzahl in der Primfactorenzerlegung einer ungeraden vollkommenen Zahl, J. Reine Angew. Math. 186 (1949), 25-29.

[28] M. Kishore, Odd perfect numbers not divisible by 3 (II), Math. of Comp. 40 (1983), 405-411.

[29] P. P. Nielsen, An upper bound for odd perfect numbers, Int.: Elec. J. Comb. Num. Th. 3 (2003), A14.

[30] P. P. Nielsen, Odd perfect numbers have at least nine distinct prime factors, Math. of Comp. 76 (2007), 2109-2126.

[31] S. L. Padwa, Nobody is even perfect - solution to problem H-188 (proposed by Raymond E. Whitney), Fib. Qtly. 10 (1972), 631.

[32] B. Peirce, On perfect numbers, N. Y. Math. Diary 2 XIII (1832), 267-277.

[33] C. Pomerance, Odd perfect numbers are divisible by at least seven distinct primes, Acta Arith. XXV (1974), 265-300. 
[34] C. Pomerance, The second largest divisor of an odd perfect number, Math. of Comp. 29 (1975), 914-921.

[35] C. Pomerance, Multiply perfect numbers, Mersenne primes, and effective computability, Math. Ann. 226 (1977), 195-226.

[36] N. Robbins, The nonexistence of odd perfect numbers with less than seven distinct prime factors, Doctoral Dissertation, Polytechnic Institute of Brooklyn. June, (1972).

[37] M. Sayers, An improved lower bound for the total number of prime factors of an odd perfect number, Master's thesis, New South Wales Institute of Technology, 1986.

[38] C. Servais, Sur les nombres parfaits, Mathesis 8 (1888), 92-93.

[39] C. Servais, Sur les nombres parfaits, Mathesis 8 (1888), 135.

[40] R. Steuerwald, Verschärfung einer notwendigen Bedeutung für die Existenz einer ungeraden vollkommenen Zahl, Bayer. Akad. Wiss. Math. Natur. (1937), 69-72.

[41] J. J. Sylvester, Note on a proposed addition to the vocabulary of ordinary arithmetic, Nature, XXXVII (1888), 152-153.

[42] J. J. Sylvester, On the divisors of the sum of a geometrical series whose first term is unity and common ratio any positive or negative integer, Nature XXXVII (1888), 417-418.

[43] J. J. Sylvester, Sur les nombres parfaits, Comp. Rend. CVI (1888), 403405.

[44] J. J. Sylvester, Sur l'impossibilitié de l'existence d'un nombre parfait qui ne contient pas au 5 diviseurs premiers distincts, Comp. Rend. CVI (1888), 522-526.

John H. Jaroma,

Department of Mathematics and Physics,

Ave Maria University,

Ave Maria, FL 34142, USA

john.jaroma@avemaria.edu

Received on 23 August 2008. 